\title{
Correlates and Predictors of Psychological Morbidity in HIV-Infected Persons: A Cross Sectional Study
}

\author{
O. H. Ezeh'1, C. C. Ezeh ${ }^{2}$ \\ ${ }^{1}$ Department of Community Medicine, ABU, Zaria, Nigeria \\ ${ }^{2}$ College of Agriculture, ABU, Zaria, Nigeria \\ Email: drohezeh@gmail.com
}

How to cite this paper: Ezeh, O.H. and Ezeh, C.C. (2019) Correlates and Predictors of Psychological Morbidity in HIV-Infected Persons: A Cross Sectional Study. Open Journal of Medical Psychology, 8, 57-65. https://doi.org/10.4236/ojmp.2019.84006

Received: July 22, 2019

Accepted: August 31, 2019

Published: September 3, 2019

Copyright $\odot 2019$ by author(s) and Scientific Research Publishing Inc. This work is licensed under the Creative Commons Attribution International License (CC BY 4.0).

http://creativecommons.org/licenses/by/4.0/

(c) (i) Open Access

\begin{abstract}
Introduction: HIV-infection is associated with many factors including: physical, social, cultural and psychosocial factors. Physiological changes associated with HIV infection may generate psychological morbidity. However, specific psychosocial factors contributing to psychological morbidity remain unclear. Identifying factors contributing to psychological morbidity may help in prevention and planning intervention. Aims/Objectives: 1) To identify significant predictors of psychological morbidity from specific dysfunctional coping traits/dimensions among HIV-infected persons; 2) To assess/determine the association between predictor variables (specific coping dysfunctional traits) and psychological morbidity; 3) To identify other correlates of psychological morbidity; 4) To make recommendations on how to reduce levels of dysfunctional coping and psychological morbidity. Methods: This cross-sectional descriptive study was conducted to examine data collected from 110 participants, HIV patients receiving HAART services from Ahmadu Bello University Teaching Hospital (ABUTH), Zaria, Kaduna State, Nigeria. Results: Results indicate that HIV-patients with high levels of psychological morbidity were more likely to use dysfunctional coping more frequently. Therefore, greater psychological distress and symptoms reporting may be associated with dysfunctional coping. Conclusion/Recommendation: The study recommends that specific dysfunctional coping dimensions should also be targeted in interventions designed to reduce psychological morbidity in HIV patients.
\end{abstract}

\section{Keywords}

Psychological Morbidity, Predictors, Correlates,

Coping Dysfunction 


\section{Introduction}

Globally, HIV continues to be a major public health problem. In 2017 NACA (National Agency for the Control of AIDS) [1], reported that Nigeria had the second largest HIV epidemic in the world with 2.8 percent HIV prevalence among adults. However, UNAIDS and NACA [2] estimate that there are 1.9 million people living with HIV in Nigeria. Viral suppression is a very important outcome of HIV treatment because when persons living with HIV are virally suppressed they remain healthy and transmission of the virus is prevented [1]. Psychosocial factors may play significant roles in the progression of HIV-infection. Psychological morbidity and dysfunctional coping are parts of psychosocial influences relating to disease progression. According to NAIIS (National HIV/ AIDS Indicator and Impact Survey) [3], the viral suppression at the national level among people living with HIV aged 15 - 49 years stands at 42.3 percent (45.3 percent among women and 34.5 percent among men). The findings of NAIIS also indicate that a total of 11.3 million adults were counseled and tested for HIV in 2016.

Coping strategies are generally defined as the behaviors, emotions, and thoughts an individual uses to adjust to the changes that occur in their life. Coping involves cognitive, affective and behavioral responses to perceived stressor. Functional coping provides protective factor. It may dissipate feelings of distress and anxiety. Psychological morbidity or psychological distress is a negative affect without the individual necessarily meeting the criteria for psychiatric disorder. Study [4] indicates that psychological morbidity was highly prevalent among patients with chronic illnesses generally and HIV infection in particular, affecting more than 40 percent of persons living with HIV-infection. This may have significant influence on HIV-spread and progression. Psychological morbidity has also been attributed to many risk factors. Dysfunctional coping is detrimental ways of coping, for example, use of drugs or alcohol, self-blame, behavioral disengagement, denial etc. Previous research [5], has shown that both psychological morbidity and dysfunctional coping were under-recognized and under-treated. Undiagnosed and untreated psychological morbidity may lead to poor physical and psychological health outcomes, for example, poor adherence to HAART services, faster disease progression, transmission and spread of the HIV-infection. Yet these factors are treatable with evidence-based psychological intervention, and may be among modifiable risk factors for prevention, and may also be amenable to psychological intervention, which would help to plan appropriate interventions to better control psychological morbidity and dysfunctional coping. Despite these adverse psychological and physical health consequences, dysfunctional coping and psychological morbidity remain undiagnosed and untreated in HIV-infected persons especially in resource-restricted countries. Functional and adaptive coping has been shown to be associated with positive and favorable HIV outcomes [5]. For example, lower negative religious coping scores and social support have been shown to predict high HAART adherence scores [6]. To 
successfully cope with a stressful situation may require the individual's ability to do something practical about it. Effective and adequate coping with HIV-infection would ultimately improve the survivors' quality of life.

Increasingly, research [4] supports psychological morbidity as a risk factor for both physical and psychological health problems, few if any study has been able to identify it's correlates and predictors, in order to plan adequate and effective intervention, treatment and prevention in chronically-ill patients.

The study identified correlates and predictors of psychological morbidity from four specific dysfunctional coping traits/dimensions, in order to inform targeted psychological screening and intervention protocols for HIV-infected persons.

\section{Research Questions}

1) Could dysfunctional coping be one of the risk factors associated with psychological morbidity?

2) What percentage of psychological morbidity is attributable to specific dysfunctional coping traits/dimensions?

3) What are the key contributions of specific dysfunctional coping traits to psychological morbidity?

4) How does dysfunctional coping contribute to psychological morbidity?

The study was aimed to examine these questions in HIV patients receiving HAART services in a hospital out-patient clinic. HIV patients may be at risk for increased psychological distress because of the bodily and psychological changes that may be generated by the HIV-infection.

\section{Objectives}

1) To examine predictors of psychological morbidity among HIV-infected persons

2) To determine association between specific dysfunctional coping traits and psychological morbidity.

3) To examine/assess the relative contributions of specific dysfunctional coping dimensions/traits to psychological morbidity.

Examining correlates and predictors of psychological morbidity in a population of HIV-infected persons would assist in planning appropriate interventions that may decrease psychological distress, promote psychological health, slow down HIV progression and increase productivity.

\section{Methods}

The 12-item General Health Questionnaire (GHQ) [7] was administered to assess participants' psychological health. Psychological morbidity was measured on a Likert Scale of 0 - 5, where 0 was no distress and 5 extremely distressed. The GHQ had demonstrated good validity and reliability [4]. Brief-COPE (28-item) was also administered to assess dysfunctional coping. Brief-COPE [8] was designed to measure coping strategies, containing both functional and dysfunc- 
tional coping items. Dysfunctional coping was defined as scoring above the mean score in the subscales. The same applied to GHQ scores.

Participants were 110 patients, 35 males (31.82 percent); 75 females (68.18 percent), mean age 39.79, SD 11.61, who were receiving HAART (highly active anti-retroviral) services at Ahmadu Bello University Teaching Hospital (ABUTH), Zaria, Nigeria selected through half-hazard/convenience sampling technique. Only subjects who were available and who were willing to participate actively in the study were selected. A total of 128 sets of questionnaires were distributed and 110 were completely returned giving 85.9 percent response rates. Participants were given information on the survey and their consent to participate in the study was obtained. Ethical clearance was also obtained from ethical committee of the hospital. The subjects were asked to indicate what they usually do when they experience a stressful event, on a 4-point scale ranging from "I don't do this at all" to "I do this a lot". The results were calculated as the sum of responses on a particular sub-scale. The results obtained on subscales of dysfunctional coping were taken into account separately and in combination of the 4 dysfunctional subscales (integrated dysfunctional coping) used in this study for data analyses. The 4 subscales include:

1) Denial (DN)

Avoidance of the problem, refusal to believe that there is problem. Activities that aid denial include, drinking, sleeping, isolation etc

2) Behavioral Disengagement (BD)

This involves giving up the attempt to deal with a situation.

3) Substance Use (SU)

This means frequent use of alcohol or other substances to cope with stress.

4) Self-Blame (SB)

Internalizing the problem and blaming oneself, beyond just taking responsibility for one's actions, which may lead to low self-esteem, or even depression etc.

\section{Results}

Data were analyzed using STATA software, version 13.1. The relationship between dysfunctional coping dimensions and psychological morbidity was analyzed using Pearson Product Moment correlation, and the contributions of dysfunctional coping dimensions to psychological morbidity were identified with multiple regression analyses. Dysfunctional coping was broken down into its' specific dimensions or traits. Although the whole 28-item Brief-COPE [8], was administered, statistical analyses were restricted to only four subscales used to measure dysfunctional coping in this study. Both multiple regression analyses and Pearson-product correlational analyses were conducted to examine the contributions of specific dysfunctional coping traits to-and their relationships with psychological morbidity. Altogether, four specific traits or dimensions of dysfunctional coping were evaluated; Denial (DN), Behavioral disengagement (BD), 
Substance use (SU), and Self-blame (SB). Following the correlational analyses, regression analyses were also carried out to examine specific dysfunctional coping traits as predictors of psychological morbidity. The four specific traits were regressed against psychological morbidity (PSYM) in HIV-infected persons.

Table 1 indicates that the majority of the patients were within the demographic group of 36 - 45 years, (38.18 percent).

Table 2 indicates that majority of the patients were within the HIV-infection duration of $2-4$ years $(38 ; 34.55$ percent) and $5-10$ years $(38 ; 34.55$ percent).

Table 3 indicates differing means for DN (5.75), BD (3.25) SU (2.54) SB (3.62) and TOT (15.16).

Data (Table 4) suggest that prevalence rates of dysfunctional coping dimensions (DN, 61.8 percent; BD, 41.8 percent; SU, 21.8 percent; SB, 55.5 percent), and that of Integrated/whole dysfunctional coping (TOT), 44.5 percent were generally high.

Table 5 indicates that psychological morbidity was positively related to substance use coping ( $\mathrm{SU}, \mathrm{P}<0.03$ ) and self-blame coping ( $\mathrm{SB}, \mathrm{P}<0.01$ ), and negatively related to behavioral disengagement coping $(\mathrm{BD}, \mathrm{P}<0.38)$, although this did not reach significant level. No significant correlation with Denial (DN, P $0.22)$.

Table 6 indicates that frequent use of self-blame (SB) was associated with the largest burden of psychological morbidity $(\mathrm{P}>0.001)$, followed by Denial (DN, $\mathrm{P}>0.01)$, and substance use (SU, P $>0.01)$.

Table 1. Distribution of respondents by age.

\begin{tabular}{cccc}
\hline Age Category & Frequency & Percent & Cumulative \\
\hline $15-25$ & 15 & 13.64 & 13.64 \\
$26-35$ & 24 & 21.82 & 35.46 \\
$36-45$ & 42 & 38.18 & 73.64 \\
$46-55$ & 18 & 16.36 & 90.00 \\
$56-65$ & 11 & 10.00 & 100.00 \\
Total & 110 & 100 & \\
\hline
\end{tabular}

Field Survey; Ezeh, 2018.

Table 2. Distribution of Respondents by Duration of HIV-infection (Survivability).

\begin{tabular}{cccc}
\hline Duration & Frequency & Percent & Cumulative \\
\hline$<1$ year & 11 & 10.00 & 10.00 \\
2 - 4 years & 38 & 34.55 & 44.55 \\
5 - 10 years & 38 & 34.55 & 79.10 \\
$>10$ years & 23 & 20.91 & 100 \\
\hline
\end{tabular}

Field Survey; Ezeh, 2018. 
Table 3. Summarizing dimensions of dysfunctional coping and their composite (total)/ integrated dysfunctional coping.

\begin{tabular}{cccccc}
\hline Variable & Obs. & Mean & Std. Deviation & Min. & Max. \\
\hline DN & 110 & 5.75 & 1.89 & 2 & 8 \\
BD & 110 & 3.3 & 1.52 & 2 & 8 \\
SU & 110 & 2.5 & 1.16 & 1 & 8 \\
SB & 110 & 3.63 & 1.51 & 2 & 8 \\
$\begin{array}{c}\text { TOTAL (Integrated } \\
\text { dysfunctional coping) }\end{array}$ & 110 & 15.16 & 3.03 & 8 & 23 \\
\hline
\end{tabular}

Field Survey; Ezeh, 2018.

Table 4. Differing prevalent rates of dimensions of dysfunctional coping and the integrated whole (Tot.) dysfunctional coping.

\begin{tabular}{ccccc}
\hline Variable & Male & Female & Prevalence Rates & Percent N = 110 \\
\hline DN & 20 & 48 & 68 & 61.8 percent \\
BD & 14 & 31 & 46 & 41.8 percent \\
SU & 13 & 11 & 24 & 21.8 percent \\
SB & 18 & 43 & 61 & 55.5 percent \\
Integrated/TOT & 17 & 32 & 49 & 44.5 percent \\
Dysfunctional Coping & & & &
\end{tabular}

Field Survey; Ezeh, 2018.

Table 5. Correlations of PSYM with DN, BD, SU, and SB.

\begin{tabular}{ccc}
\hline Variables & Correlations & Significance Values \\
\hline DN & 0.1201 & 0.22 \\
BD & -0.0855 & 0.38 \\
SU & 0.2067 & $0.03^{*}$ \\
SB & 0.2515 & $0.01^{*}$ \\
\hline
\end{tabular}

Field Survey; Ezeh, 2018.

Table 6. Regress PSYM against DN, BD, SU, and SB.

\begin{tabular}{cccc}
\hline PSYM & Coefficient & Standard Error. & P> \\
\hline DN & 0.1901533 & 0.066903 & $0.01^{\star}$ \\
BD & -0.0667384 & 0.1136616 & 0.558 \\
SU & 0.4190917 & 0.1473752 & $0.01^{\star}$ \\
SB & 0.3865439 & 0.1174042 & $0.001^{\star}$
\end{tabular}

$R^{2}=0.7650$. Field Survey; Ezeh, 2018.

Overall, results revealed that correlations of dysfunctional coping dimensions with psychological morbidity yielded; $\mathrm{DN}, \mathrm{R}=0.1201, \mathrm{P}=0.22, \mathrm{BD}, \mathrm{R}=$ $-0.0855, \mathrm{P}=0.38, \mathrm{SU} \mathrm{R}=0.2067, \mathrm{P}=0.03^{*}, \mathrm{SB}, \mathrm{R}=0.2515, \mathrm{P}=0.01^{*}$. 
The results also revealed that, the use of some specific traits of dysfunctional coping (SU, P > 0.01; SB P > 0.001) predicted psychological morbidity, in HIV-infected persons. In other words total/combined/integrated coping dysfunction predicted psychological morbidity. Regression analyses indicate that with the /exception of $\mathrm{BD}$, all the dysfunctional coping dimensions predicted psychological morbidity, although Self-blame emerged as the strongest predictor of psychological morbidity, among the specific dysfunctional coping dimensions. Self-blame is therefore the strongest clinical risk factor for psychological morbidity in this study.

\section{Discussion}

The findings revealed that more than 49 (44.5 percent) HIV-patients were dysfunctional in their coping with HIV-associated stresses. The overall prevalence rates of coping dysfunction and psychological morbidity among the patients were 44.5 percent and 61 percent respectively.

Psychological morbidity and dysfunctional coping are part of psychosocial influences relating to disease progression [9]. When psychological problem or difficulty is present, it interferes with effective and adequate disease management.

Specific dysfunctional coping traits/dimensions contributing to psychological morbidity were identified by the study. Interventions to address dysfunctional coping in HIV-infected persons would also improve psychological morbidity and psychological functioning. Effective and adequate social support and functional coping may dissipate feelings of distress.

The study suggests that dysfunctional coping was associated with high levels of psychological morbidity, and also predicted it (psychological morbidity). This finding is line with other study that had shown that HIV-infected individuals with high levels of perceived stress typically had poorer stress management skills and less appropriate coping mechanism [4] [10].

Several implications can be derived from the findings of this study;

Many HIV-infected persons experience severe psychological difficulties that may require intervention. This means there is need for routine psychological screening at outpatient contacts. Untreated psychological conditions may escalate and result to job and other issues.

Yet these factors may be modifiable by psychological interventions, for example social support can mitigate psychological distress since chronically ill patients with greater social support show better psychological adjustment [11].

Dysfunctional coping was found to be a risk factor for psychological morbidity therefore, functional, effective and adequate coping would constitute protective factors. This finding is line with other study that reports poor stress management skills in HIV-infected persons [12].

Other correlates of psychological morbidity that may incline HIV-infected persons to be dealing with stress by means of dysfunctional coping may include; low self-esteem, poor utilization of psychological screening tools and support 
services or total lack of support services. All these may constitute risk factors for dysfunctional coping and psychological morbidity.

Functional coping may provide health protective factor. Other factors that may be protective against development of dysfunctional coping and psychological morbidity include; personality factors, resilience, adequate utilization of social support services and other health-protective behaviors, etc.

Dysfunctional coping contributes to decline in psychological health and quality of health. Coping dysfunction may constitute secondary stress to those who use them frequently.

\section{Conclusion}

The study has been able to demonstrate that specific dysfunctional coping dimensions significantly contributed to psychological morbidity. Psychological impact of dysfunctional coping increased the risk of psychological morbidity. Therefore reducing levels of dysfunctional coping would also reduce psychological morbidity. Interventions to address psychological morbidity should also target dysfunctional coping in order to improve psychological functioning and overall quality of life in HIV-infected persons.

\section{Recommendation}

The results of the tests may help determine the best coping skills intervention for HIV-patients coping by means of frequent use of dysfunctional coping strategies. Clinical interventions can be developed to enhance coping skills, and improve survival rates of persons living with HIV-infection. The findings provide new information on the role of psychosocial issues in the spread and treatment of HIV. Screening tools and counseling interventions to reduce and prevent the risks of psychological health problems should be employed.

Effective and adequate screening and treatment of dysfunctional coping and psychological morbidity in HIV-infected persons, insight-oriented counseling, motivational interviewing, and other supportive services to address these psychological issues were strongly recommended.

\section{Conflicts of Interest}

The authors declare no conflicts of interest regarding the publication of this paper.

\section{References}

[1] NACA (2017) National Strategic Framework on HIV and AIDS: 2017-2021.

[2] UNAIDS and NACA (2019) UNAIDS Report. HIV/AIDS in Nigeria. http://pushingforthelastmile.com

[3] NAIIS (2018) National HIV/AIDS Indicator and Impact Survey. http://www.internationalhealthpolicies.org

[4] Ezeh, O.H. (2017) Socio-Demographics of HIV-Infected Persons with Psychological Morbidity in Zaria, Nigeria. Open Journal of Medical Psychology, 6, 228-232. 
https://doi.org/10.4236/ojmp.2017.64018

[5] Ezeh, O.H. (2019) Diagnosing Dysfunctional Coping in HIV-Infected Persons. Open Journal of Medical Psychology, 8, 36-43. https://www.scirp.org

[6] Dalmida, S.G., McCoy, K., Koenig, H.G., Miller, A., Holstad, M.M. and Thomas, T.L. (2018) Correlates and Predictors of Medication Adherence in Out-Patients Living with HIV/AIDS. Journal of HIVI AIDS and Social Services, 17, 402-420.

https://www.tandfonline.com https://doi.org/10.1080/15381501.2018.1502709

[7] Goldberg, D. (1992) The General Health Questionnaire (GHQ-12). NFER-Nelson, Windsor.

[8] Carver, C.S. (1997) You Want to Measure Coping Protocol Too Long: Consider the Brief Cope. International Journal of Behavioral Medicine, 4, 92-100. https://doi.org/10.1207/s15327558ijbm0401_6

[9] Balbin, E.G. (2000) Stress and Coping: The Psychoneuroimmunology of HIV/AIDS. Best Practice Research: Clinical Endocrinology and Metabolism, 13, 615-633. https://doi.org/10.1053/beem.1999.0047

[10] O'Cleirigh, C. and Safren, S. (2007) Breaking the Mold or Business as Usual? Meeting the Challenges of HIV Prevention in People with Serious Mental Illness and Substance Use Disorders. Clinical Psychology, Science and Practice, 14, 34-38. https://doi.org/10.1111/j.1468-2850.2007.00060.x

[11] Aneshensel, C.S. and Stone, J.D. (1982) Stress and Depression: A Test of Buffering Model of Social Support. Archive of General Psychiatry, 39, 1392-1396. https://doi.org/10.1001/archpsyc.1982.04290120028005

[12] Joseph, R.S. (2016) The Importance of Stress Management for People with HIV/ AIDS. GoodTherapy.org. https://www.goodtherapy.org 